\title{
Evaluación de la eficiencia de extractos naturales en el proceso de coagula- ción floculación de aguas crudas, con fines de potabilización
}

\author{
Evaluation of the efficiency of natural extracts in the coagulation floculation process of raw \\ water, for purification purposes
}

Carlos Augusto Benjumea-Hoyos, Manuela Toro Martínez, Valerya Luna Marin

DOI. 10.21931/RB/2021.06.02.17

Resumen: El presente estudio permitió por medio de ensayos de jarras, evaluar la eficiencia de los extractos naturales de Zea mays (Maíz), Aloe vera (Sábila) y Citrus sinensis (Naranja) para disminuir los valores de turbidez en aguas crudas con fines de potabilización y su efecto en el pH y la conductividad. Esto con el fin de investigar alternativas viables que permitan mejorar la calidad del agua cruda y que sean de fácil acceso para comunidades marginales; el uso de extractos naturales en el proceso de potabilización de aguas y en específico en la remoción de turbidez permite evaluar la capacidad de estos en relación con los compuestos químicos convencionales utilizados en dicho proceso. En este sentido, se obtuvo una remoción en la turbidez del 85\% con los extractos de maíz y sábila; y del 69\% con el extracto de naranja. Los resultados obtenidos dejan ver los beneficios de utilizar extractos naturales en los procesos de tratamiento de aguas, el fácil acceso a la materia prima requerida, su bajo costo, producción mínima de lodos inocuos debido a la no presencia de sales metálicas y su aporte a reducir el uso de coagulantes químicos, serían aportes importantes en la disminución de impactos ambientales asociados a los riesgos que este tipo de lodos convencionales podrían provocar.

Palabras clave: Coagulación, floculación, extractos naturales, turbidez, pH, conductividad, calidad del agua, potabilización.

\begin{abstract}
The present study allowed, through jar tests, to evaluate the efficiency of the natural extracts of Zea mays (Corn), Aloe vera (Aloe vera), and Citrus sinensis (Orange) to reduce the turbidity values in raw water for purification purposes and its effect on $\mathrm{pH}$ and conductivity. This is to investigate viable alternatives that improve the quality of raw water and are easily accessible to marginal communities; the use of natural extracts in the water purification process and specifically in the removal of turbidity allows evaluating their capacity concerning the conventional chemical compounds used in the process. In this sense, a turbidity removal of $85 \%$ was obtained with the extracts of corn and aloe; and 69\% with the orange extract. The results obtained show the benefits of using natural extracts in drinking water treatment processes, the easy access to the required raw material, its low cost, minimum production of harmless sludge and its contribution to reducing chemical coagulants' use would be contributions significant in reducing environmental impacts associated with the risks that this type of conventional sludge could cause.
\end{abstract}

Key words: Coagulation, flocculation, natural extracts, turbidity, $\mathrm{pH}$, conductivity, purification.

\section{Introducción}

Según UNICEF $(2019)^{1}$, con base en la problemática del acceso al agua potable, se estima que 1 de cada 10 personas todavía carecen de servicios básicos, incluidos los 144 millones que beben agua de superficie que no ha recibido tratamiento. Además, evidencia que 8 de cada 10 personas que viven en zonas rurales carecen de acceso al agua potable, y en 1 de cada 4 países con estimaciones para diferentes grupos de riqueza, la cobertura de los servicios básicos era más desfavorecida para personas de escasos recursos económicos, como consecuencia de la mala gobernabilidad y gobernanza del agua en todo el mundo ${ }^{2}$. A lo anterior se le suma la alta demanda de agua que aumentará entre un 20 y $30 \%$ en 2050 respecto al nivel actual, por el crecimiento poblacional, desarrollo económico y los modos de consumo ${ }^{3}$.

En el caso de Colombia a pesar de que los servicios públicos constituyen un derecho humano fundamental que debe proveerse de manera obligatoria a la comunidad en las cantidades mínimas ${ }^{4}$, esto no se ha podido satisfacer en todo el territorio Nacional, lo cual hace más evidente la falta de gobernabilidad y gestión ${ }^{5}$. En Consecuencia, 28.1 millones de personas en la zona urbana cuentan con agua potable, es decir $86.11 \%$ de la población total y alrededor de 3.8 millones de

personas reciben agua no apta para consumo humano, haciendo referencia a un $\mathbf{1 1 . 5 6 \%}$ de la población total del país ${ }^{6}$. Los municipios con menor cobertura de agua potable presentan consecuencias mortales en la población por lo que por cada 100.000 nacidos hay 96 muertes maternas asociadas a la falta de cobertura de acueducto y alcantarillado, además, 11 de cada 100.000 menores de 5 años mueren a causa de enfermedades diarreicas ${ }^{7}$.

Los cuerpos de agua cruda contienen partículas coloidales que generan turbidez debido a sedimentos y nutrientes, los cuales no precipitan completamente de manera natural y, por ende, deben ser removidos ${ }^{8,9}$. Por tal motivo, la clarificación del agua, que se entiende como el retiro de materiales sólidos y coloidales, es fundamental en el proceso de potabilización, que incluye las fases de coagulación-floculación en el cual las partículas presentes se aglomeran formando pequeños gránulos con un peso específico mayor; de esta forma las partículas sedimentan y ocurre la remoción de los materiales en suspensión, lo que permite que el agua alcance características físicas y químicas idóneas como un paso en su tratamiento, con el fin de lograr que esta cumpla las normas y estándares de salud pública ${ }^{10}$. 
Generalmente en las plantas de potabilización se utilizan coagulantes inorgánicos, muchos de ellos como el sulfato de aluminio y cloruro férrico, entre otros, que se han utilizado para eliminar las impurezas y las partículas coloidales presentes en los cuerpos de agua debido a su rendimiento y disponibilidad ${ }^{11}$. Sin embargo, existe evidencia que relaciona a los coagulantes a base de aluminio, con el desarrollo de enfermedades como el Alzheimer en los seres humanos ${ }^{12-15}$, así mismo con la producción de grandes cantidades de sedimentos que, al ser vertidos en las fuentes de agua, se convierten en un problema ambiental debido a que en altas dosis pueden llegar a ser tóxico ${ }^{16}$

Ante esta problemática, se hace necesaria la búsqueda de alternativas que puedan sustituir total o parcialmente las sales de hierro y aluminio, así como los polímeros orgánicos sintéticos ${ }^{17}$. En varios países de Latinoamérica, desde los años 70 en adelante se propuso la utilización de los coagulantes naturales, donde la mayoría de estos se derivan de semillas, hojas, cortezas o savia, raíces y frutos de árboles y plantas, además se pueden extraer de microorganismos, animales y tejidos vegetales ${ }^{18}$ con el propósito de reducir el consumo de reactivos químicos.

De acuerdo con lo anterior, en la literatura se han encontrado antecedentes sobre el uso de coagulantes naturales en Colombia para ser usados en los procesos de clarificación de agua $^{19}$ tales como el cactus Opuntia ficus, samán o árbol de Lluvia Pithecellobium ${ }^{20}$ moringa oleifera, entre otros ${ }^{21-23}$. Esto se ha estudiado principalmente en regiones del caribe, debido a que demuestran que no es posible acceder al agua potable en las comunidades de escasos recursos económicos y, en especial la población rural ${ }^{24}$.

Ante este panorama surge la necesidad de evaluar mediante este trabajo el poder coagulante de Citrus sinensis, Zea mays y Aloe vera, en el proceso de clarificación en el agua cruda.

\section{Materiales y métodos}

\section{Área de estudio}

Los ensayos se realizaron en el laboratorio de monitoreo ambiental de la Universidad Católica de Oriente ubicada en el municipio de Rionegro-Antioquia. Las muestras de agua fueron obtenidas de la quebrada el Águila con coordenadas geográficas latitud 69'6.00" N, longitud $75^{\circ} 21^{\prime} 59.45 "$ O, la cual es afluente del Río Negro (figura 1).

A las muestras se les ajustó su turbidez inicial, bajo pa- rámetros similares a los empleados por Caicedo $(2017)^{25}$, en un modelo de solución de mezcla de fondo a una relación de 1/1 de limo propio de la zona de estudio y agua cruda de la quebrada, preparando la solución en un recipiente plástico previamente lavado y realizando un mezclado de manera manual. La mezcla se dejó reposar con el fin de lograr el asentamiento de los sólidos sedimentables, y al mismo tiempo se permitió el aumento de la turbidez hasta garantizar rangos de 255 a 309 NTU. Se determinaron las variables fisicoquímicas de la mezcla como $\mathrm{pH}$ (Unidades de $\mathrm{pH})$, conductividad $(\mu \mathrm{S} / \mathrm{cm})$ y concentración de cloruro de sodio $\mathrm{NaCl}(\mathrm{mg} / \mathrm{L})$ con un multiparamétrico marca HACH hq40d, modelo MULTI 340i y la turbidez con un turbidímetro marca HACH 2100q, método nefelométrico (método 2130B), ambos calibrados con soluciones estandarizadas.

En la preparación de los extractos naturales, se inició con el tratamiento del material vegetal. Para el caso de Citrus sinensis (Naranja) se recolectaron las cascaras y se eliminó el bagazo (Mesocarpio), se dejó secar durante 24 horas para lograr una humedad de alrededor del 10\%, luego se trituró en trozos de $0.5 \mathrm{~mm}$ aproximadamente de manera manual y mecánica; se realizó el mismo proceso con el Zea mays (Maíz) a diferencia de que la materia prima de este último se obtiene seca.

Finalmente, para el material vegetal de Aloe vera (Sábila) se utilizó solo el cristal interno que esta contiene, dado que este representa hasta un $80 \%$ del peso total de la planta ${ }^{26}$. Con el fin de evitar la pérdida de las propiedades originales del Aloe vera, no se procedió a un secado, sino que se sometió a un macerado hasta lograr un cambio de estado de sólido a semilíquido.

Para la extracción del componente activo, se preparó una solución al 5\% (p/v) bajo precipitación con $100 \mathrm{ml}$ de alcohol etílico $(90 \% \mathrm{v} / \mathrm{v})$ por cada 5 gramos de material vegetal obtenido, dicha solución se mezcló con agitadores magnéticos durante 30 min y un tiempo de sedimentación de 1 hora. Posteriormente se separó el sólido y liquido de la solución con una bomba de vació y filtros de papel. La porción sólida obtenida se llevó a secado en horno a $70^{\circ} \mathrm{C}$, solo para el caso de la naranja y el maíz.

Seguidamente, se procedió a la preparación del extracto natural para ser utilizado como coagulante. Para ello, se prepararon disoluciones al $5 \%(\mathrm{p} / \mathrm{v})$ compuestas por 5 gramos de los sólidos secos por cada $100 \mathrm{ml}$ de una solución salina de $\mathrm{NaCl}$ a concentraciones de 0.125 M, 0.25 M y 0.5 M. Dichas
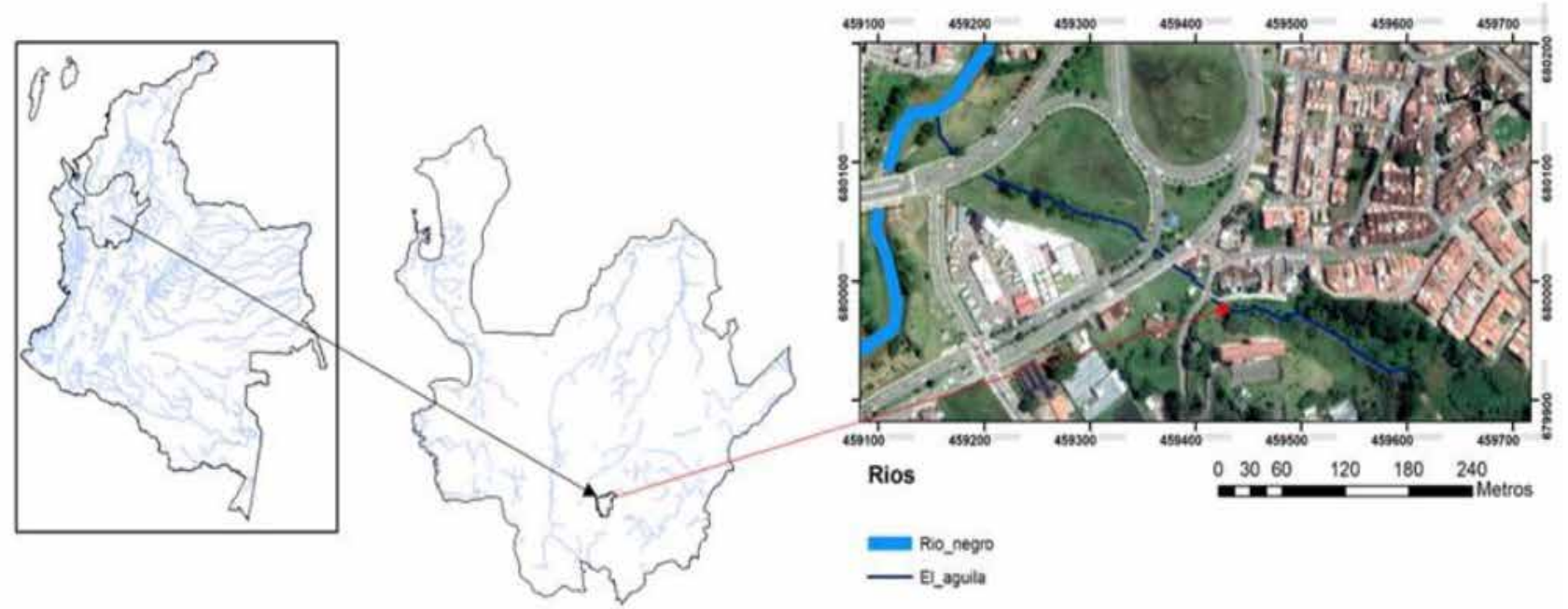

Figure 1. Localización punto de muestreo Quebrada El Águila, Rionegro-Antioquia. Fuente: Autores. 
disoluciones se mezclaron durante 30 minutos con agitadores magnéticos, y una vez transcurrido este tiempo se filtraron con la bomba de vació y papel filtro. El líquido filtrado constituye el extracto crudo salino (ECS) en sus tres distintas concentraciones $^{27,28}$

Los ECS fueron utilizados en los ensayos de actividad coagulante, con ayuda del método conocido como test de jarras según lo establecido por la ASTM $(2003)^{29}$ y lo propuesto por Norma Técnica Colombiana NTC 3903 en cuanto al procedimiento para el ensayo de coagulación-floculación en un recipiente con agua o método de jarras de manera individual. El procedimiento se realizó con cinco beacker de $1000 \mathrm{ml}$ a los cuales se les adiciono el agua cruda, dejando uno como blanco (Control) al cual no se le adiciono ningún extracto; los otros cuatro se dosificaron con $7,10,12$ y $15 \mathrm{ml}$ respectivamente. Las anteriores soluciones se sometieron a una mezcla rápida a 150 revoluciones por minuto (rpm) durante 3 min, seguida de una mezcla lenta a 25 rpm durante 20 min, por último, se finalizó con la fase de sedimentación durante 20 min y se midieron nuevamente las variables fisicoquímicas de cada uno de los beackers.

Para determinar el efecto de diferentes tipos de coagulantes (Citrus siniensis, Zea mays y Aloe vera) sobre las variables $\mathrm{pH}$, conductividad y porcentaje de remoción de turbiedad se hizo uso de un diseño unifactorial con tres réplicas para cada uno de los tratamientos utilizados.

Con el fin de establecer diferencias estadísticas significativas entre los tratamientos se hizo uso de un análisis de varianza ANOVA con un 95\% de confianza seguido de un análisis post-hoc Tukey-HSD con la misma confianza. Los supuestos de normalidad de los residuales se verificaron por medio de la prueba de Kolmogorov-Smirnov ${ }^{30}$ y Shapiro \& Wilk (1965) ${ }^{31}$ y la homocedasticidad de los residuales por medio de la prueba de Levene $(1960)^{32}$. Los resultados se presentaron a partir de una gráfica de interacción de medias entre uno o dos factores. Todo el componente estadístico se desarrolló en el software RWizard $4.2^{33}$.

Teniendo en cuenta lo establecido en la metodología, la cual se encuentra enfocada en evaluar la actividad coagulante de los extractos naturales en base salina (ECS), los tratamientos analizados como Control, a los cuales no se les realizo adición de extracto coagulante, no se consideraron dentro del tratamiento estadístico debido a que estos presentaron una remoción de turbidez por sedimentación natural, la cual fue sobrepasada visualmente por la actividad coagulante de los extractos, posterior a los 10 primeros minutos de comenzada la metodología establecida con el test de jarras.

\section{Resultados}

La tabla 1 presenta la caracterización de las variables fisicoquímicas evaluadas en el agua cruda realizada durante el presente estudio. Se estableció un intervalo de oscilación para cada variable teniendo en cuanta las repeticiones realizadas.

Con la finalidad de encontrar el mejor extracto por su acción coagulante, se estimó de manera individual para cada uno cuál fue su mejor tratamiento con base a la concentración salina de $\mathrm{NaCl}$ de este.

El análisis de varianza ANOVA permitió conocer la relación del factor concentración $(\mathrm{mg} / \mathrm{L})$ de $\mathrm{NaCl}$, sobre la variable respuesta porcentaje de remoción de turbidez con cada uno de los tres extractos coagulantes utilizados, esta se realizó con una confiabilidad del 95\%. Se tuvo en cuenta el valor de P, que para los tres extractos presento valores menores de 0.05 indicando que existen diferencias significativas entre los tratamientos evaluados en cuanto a el porcentaje de remoción de turbidez final obtenida.

El porcentaje de remoción de turbidez en relación con el extracto aplicado se muestra en la figura 2, donde teniendo en cuenta la turbiedad inicial del agua cruda, se observó y determino que los tratamientos con mayor eficiencia por extracto fueron a una concentración de 0.5 molar de $\mathrm{NaCl}$, obteniendo un porcentaje de remoción de turbidez de hasta un 85\% para el caso del maíz y la sábila, mientras que para el extracto de la naranja se alcanzó una de remoción de hasta un 69\%.

Lo anterior constituyen los resultados principales de la presente investigación, por lo cual se analizarán seguidamente solo los tratamientos que presentaron mayor eficiencia en la remoción de turbidez en cada caso, para ser relacionados con las variables fisicoquímicas después del proceso de coagulación.

Con el fin de determinar la dosis óptima de coagulante para cada extracto, se evaluó el porcentaje de remoción de turbidez por dosis adicionada en los mejores tratamientos.

Por medio nuevamente de análisis de varianza ANOVA, se encontró que los valores de $\mathrm{P}$ para todos los extractos fueron mayores de 0.05 lo que indica que no existen diferencias significativas entre la remoción de turbidez con respecto a la dosis de coagulante adicionada.

En la figura 3, se identifica que para el tratamiento con maíz a una concentración de 0.5 molar de $\mathrm{NaCl}$, la eficiencia más alta se presentó con un porcentaje de remoción de turbidez de un $85 \%$ con dosis de 7 y $12 \mathrm{ml}$, pasando de valores de turbidez de 269 NTU a 40.6 y 41.2 NTU; respectivamente. Mientras que con las dosis de 10 y $15 \mathrm{ml}$ se lograron remociones de un $83,5 \%$ con valores finales de turbidez de 44.3 y 46.9 NTU, respectivamente. Para los tratamientos con sábila a concentración de 0.5 molar de $\mathrm{NaCl}$ las dosis con mejor efecto fueron las de 10, 12 y $15 \mathrm{ml}$ con remoción hasta de un 85\%, pasando de una turbidez inicial de 300 NTU a 47.4, 48.9 y 46 NTU respectivamente, mientras que la dosis de $7 \mathrm{ml}$ mostro una disminución de la turbidez hasta 71.3 NTU. Posteriormente se encontró que, para el extracto realizado con naranja, se presentó una remoción de hasta un 69\% con la dosis de $15 \mathrm{ml}$ pasando de una turbidez inicial de 296 NTU a 87.8 NTU, mientras que las de $10 \mathrm{ml}$ y $12 \mathrm{ml}$ muestran remociones similares de hasta un 66\% pasando a remover la turbiedad hasta 96.4 NTU y 97.8 NTU respectivamente. Finalmente, la menor remoción fue con $7 \mathrm{ml}$, disminuyendo la capacidad del coagulante hasta remociones de un 63\% finalizando con una turbidez de 107 NTU.Se evaluó de manera general el efecto independiente de las variables fisicoquímicas en el diseño metodológico, con el fin de determinar cuál de las ellas tenía un efecto mayor en relación con el porcentaje de remoción de turbidez.

\begin{tabular}{lll}
\hline PARAMETRO & UNIDAD & RANGO \\
\hline Turbidez & NIU & 255 a 309 \\
\hline $\mathrm{pH}$ & Unidades de $\mathrm{pH}$ & 6.52 a 8.38 \\
Conductividad & $\mu \mathrm{S} / \mathrm{cm}$ & 54.1 a 101.9 \\
NaCl & $\mathrm{mg} / \mathrm{L}$ & 26.5 a 53
\end{tabular}

Tabla 1. Rango de oscilación de las variables fisicoquímicas de la muestra de agua. 

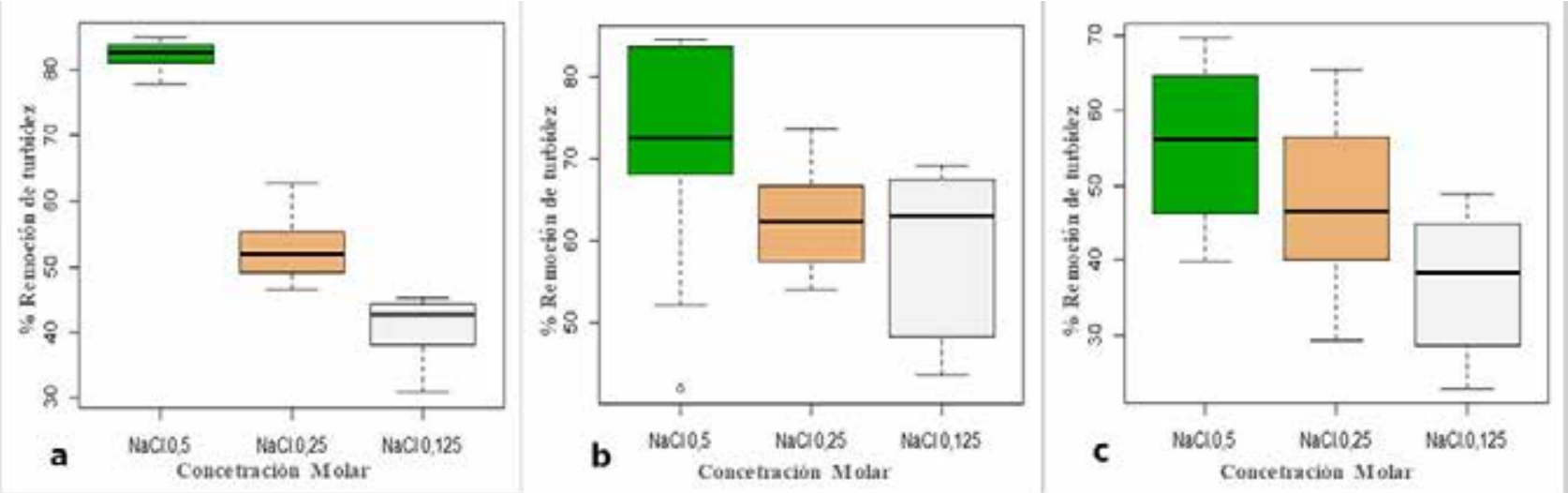

Figure 2. Porcentaje de Remoción de turbidez con extracto de Zea mays "maíz" (a), Aloe vera "sábila" (b) y Citrus sinensis "naranja" (c).
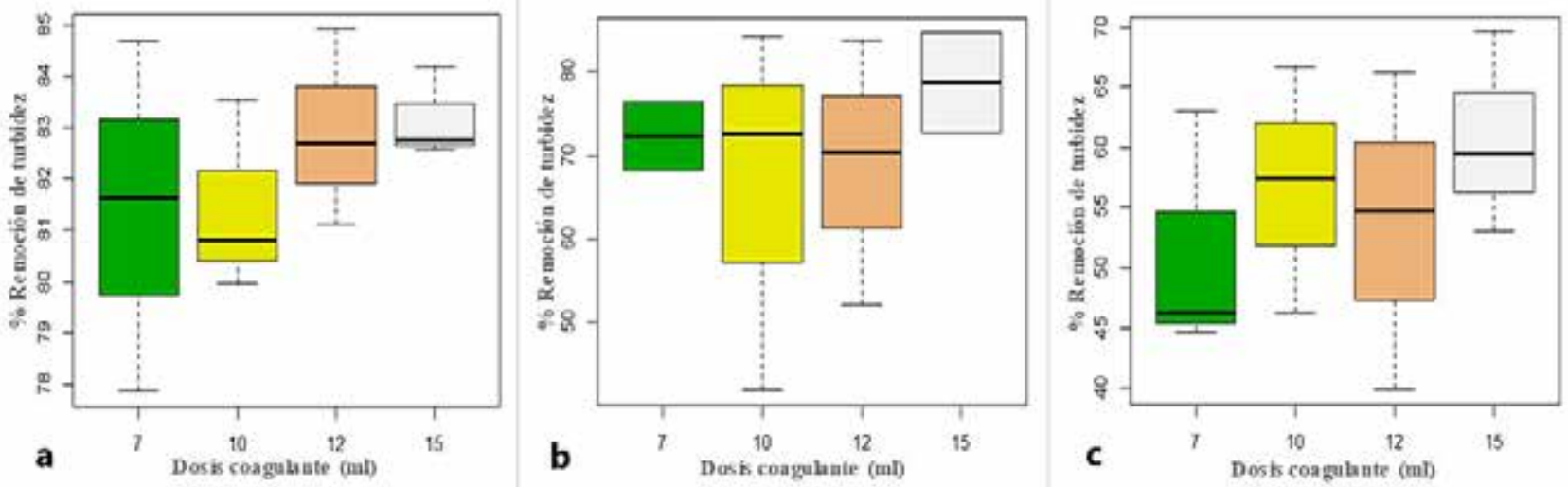

Figure 3. Porcentaje de remoción de turbidez por dosis de coagulante de extractos de Zea mays "maíz" (a), Aloe vera "sábila" (b) y Citrus sinensis "naranja" (c).

El análisis arrojo un efecto del $62 \%$ del $\mathrm{pH}$ y del $38 \%$ de la conductividad ( $\mu \mathrm{S} / \mathrm{cm})$ en los tratamientos con coagulantes naturales en el agua cruda con rangos de turbidez inicial de 255 - 309 NTU.

En la figura 4 se presenta el efecto del $\mathrm{pH}$ teniendo como factor principal las dosis de coagulantes adicionadas para el tratamiento óptimo por extracto determinado; dicha variable no presenta diferencias significativas entre tratamiento, lo que se logra visualizar dado el entrecruzamiento del error estándar (líneas verticales). Se observa que para el maíz la muestra presento un valor inicial de $\mathrm{pH} 6.95$ finalizando con un valor de 7.01 en todos los tratamientos. Con la sábila el valor inicial de $\mathrm{pH}$ fue de 6.52 concluyendo con valores de $\mathrm{pH} 6.93,6.79$, 6.67 y 6.70 para los tratamientos con dosis de 7, 10, 12 y 15 $\mathrm{ml}$ respectivamente; en tanto con la naranja este presento un valor inicial de $\mathrm{pH}$ de 6.12 variando de 7.32, 7.19, 7.15 y 7.06 con respecto a las dosis de $7,10,12$ y $15 \mathrm{ml}$ respectivamente.

En cuanto a la variabilidad de la conductividad, se puede observar en la figura 5 su relación con la dosis de coagulante adicionada y su efecto con respecto al porcentaje de remoción de turbidez en cada uno de los tratamientos. La relación entre la conductividad $(\mu \mathrm{S} / \mathrm{cm})$ y la concentración salina de $\mathrm{NaCl}$ fue directamente proporcional, las cuales presentaron un aumento progresivo en correlación con la dosis de coagulante adicionado, obteniéndose valores de hasta 1063 uS/cm para el maíz, 603.8 uS/cm para sábila y 1246 uS/cm con la naranja para la dosis de $15 \mathrm{ml}$, los cuales fueron los valores más altos, teniéndose que para las dosis más bajas esto disminuyo proporcionalmente.

\section{Discusión}

Se encontró cómo dosis óptima de ECS para el maíz el tratamiento de $7 \mathrm{ml}$, para el caso de la sábila de $10 \mathrm{ml}$ y para la naranja de $15 \mathrm{ml}$, todas a una concentración de 0.5 molar de $\mathrm{NaCl}$; esto debido a que no se presentaron diferencias significativas con las demás dosis y que además se obtuvo la mayor eficiencia en remoción de turbidez, lo cual beneficia, entre otras cosas la baja cantidad de coagulante requerido en el proceso y el poco efecto en las variables fisicoquímicas evaluadas, principalmente el pH.

Con respecto a la actividad coagulante del extracto de maíz para disminuir la turbidez, según Shogren (2009) ${ }^{34}$ el componente activo que le da la capacidad coagulante-floculante en el tratamiento de aguas es el almidón, el cual es un polímero natural que cuenta además con una alta biodegradabilidad; con dicho componente activo se ha logrado en algunos casos remociones de hasta un 90\% de turbidez y color en fuentes hídricas superficiales $^{10}$. Lo anterior, con base a los resultados obtenidos para el tratamiento más eficiente con el maíz en relación con la remoción de turbidez y la dosis optima de coagulante, coincide con lo reportado Jiménez y Piscal (2015) ${ }^{35}$, quienes consiguieron una remoción del $86.77 \%$ y afirman que los altos valores de turbidez permiten que el almidón contenido en el maíz funcione como catalizador y logre que más partículas coloidales se adhieran para formar flocs de mayor peso, promoviendo la remoción de turbidez en aguas crudas. Por su parte Aguirre et al. (2018) ${ }^{28}$ lograron obtener una remoción del 68\% y Sotheeswaran et al. $(2011)^{36}$ obtuvieron una eficiencia del 19\%; estos resultados demuestran la influencia de las diferentes metodologías aplicadas para la extracción del componente activo. 


\section{4}

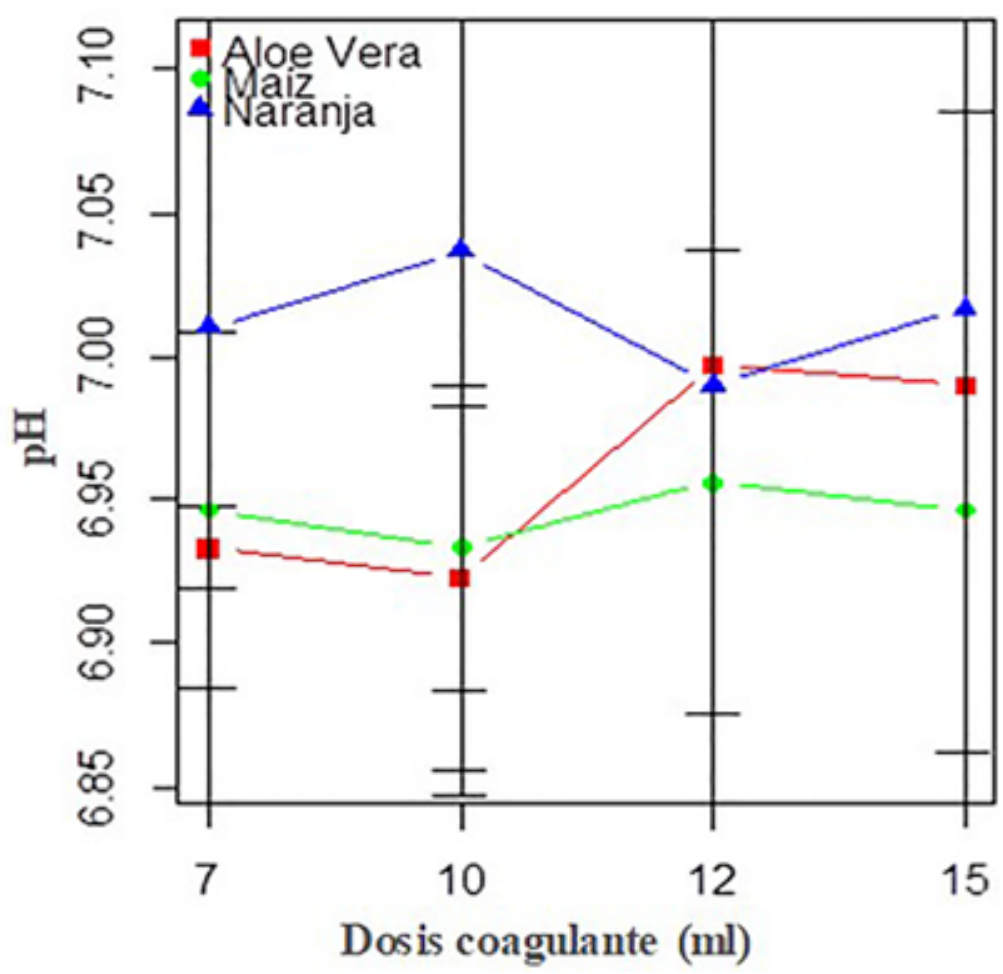

Figure 4. Variación del pH con respecto a la dosis y el extracto natural.

Figure 5. Variación de la conductividad ( $\mu \mathrm{S}$ / $\mathrm{cm})$ con respecto a la dosis y el extracto natural para los mejores tratamientos.

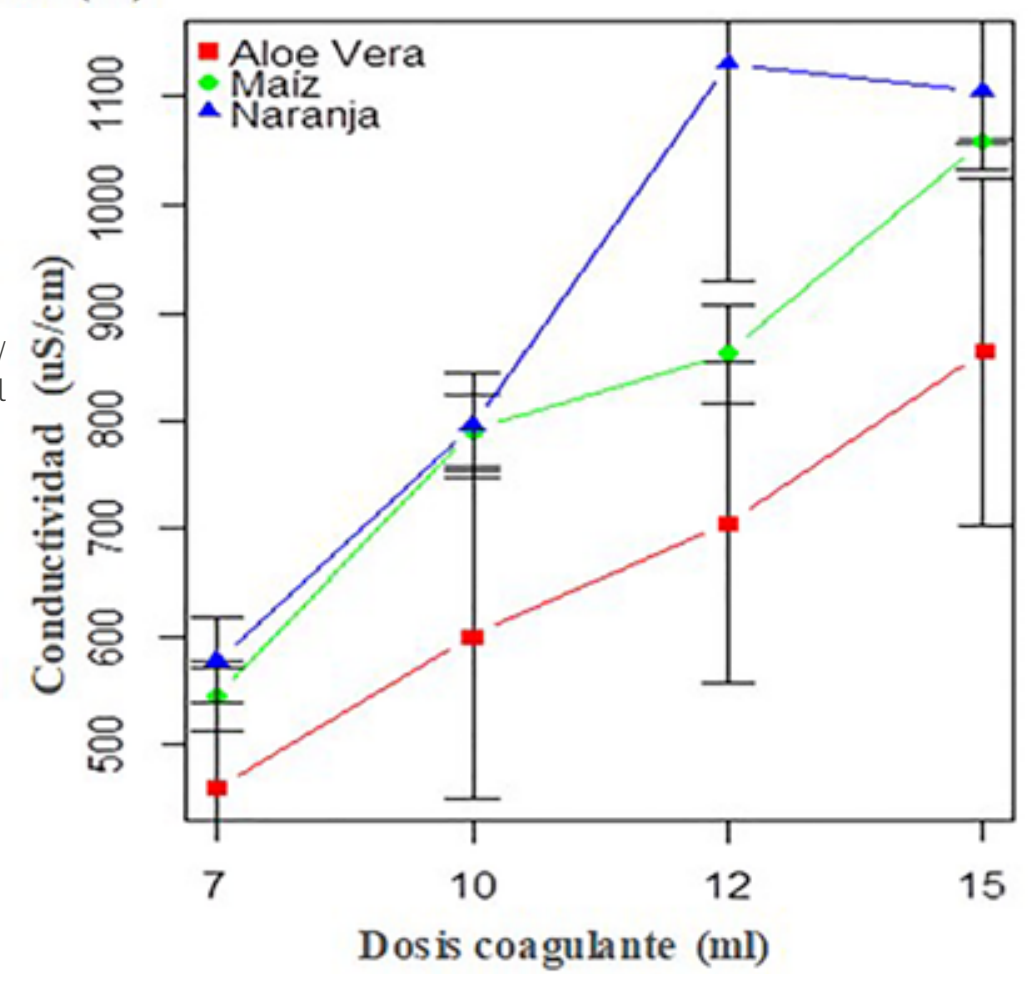

Por otra parte, la diferencia de cargas iónicas entre el almidón presente en el maíz y la poca variabilidad del pH de la muestra en relación con la dosis de coagulante aplicado en el presente estudio, generaron un equilibrio químico entre las partículas, lo que favoreció la eficiencia de remoción de turbidez y lo que se puede asociar, inclusive, a que se ha demostrado una afinidad entre dichas variables, donde se afirma que a menores dosis de coagulante y $\mathrm{pH}$ por debajo de la neutralidad se favorece el proceso de coagulación ${ }^{37}$.

La actividad coagulante del extracto de sábila y el de la naranja se encuentra ligada a la pectina ${ }^{38}$, esta se encuentra asociada a la capacidad de gelificación natural de los compuestos que a su vez permite que sea soluble en agua y a que sea eficiente para ser utilizada en procesos de tratamientos de coagulación de aguas crudas $^{39}$, donde además su capacidad coagulante se encuentra estrechamente relacionada con la disponibilidad de cationes bivalentes ${ }^{40}$.

En relación con lo obtenido con el extracto de sábila y la dosis optima de coagulante determinada para dicho tratamiento, se observa un comportamiento similar a lo reportado por Diestra y Ramos (2019) ${ }^{41}$ los cuales obtuvieron una remoción de turbidez con extractos de sábila desde un $80.98 \%$ hasta un 88.5\% con tiempos de floculación de 25 minutos, coincidiendo con lo realizado en el presente estudio, lo que además confirma 
que dicho tiempo es óptimo y evita que los flocs se destabilicen y comiencen nuevamente a aumentar los valores de turbidez. Lo primero, también se relaciona con lo propuesto por Munavalli $(2017)^{42}$ quien alcanzo remociones entre un $75 \%$ a un $81 \%$ en aguas con turbidez entre 70-90 NTU, lo que puede deberse a lo afirmado por Morales $(2018)^{43}$ donde se logró demostrar que a mayor cantidad de extracto de sábila se pueden obtener mejores resultados en la remoción de turbidez, lo que permite inferir que en el presente estudio un aumento en la concentración del extracto podría presentar remociones incluso mayores al $90 \%$.

Cabe resaltar que, en lo realizado por Mendoza, et al. $(2014)^{44}$, donde se trabajó el extracto en solución acuosa (agua destilada) solo se logró una remoción del 22.5\% confirmando que el extracto de Alo vera en solución salina logra una mayor destabilizacion de los coloides y es más efectiva para ser utilizada en los tratamientos de coagulación y floculación del agua.

Es importante mencionar que el $\mathrm{pH}$ en el tratamiento con extracto de sábila no presento variaciones significativas entre el $\mathrm{pH}$ y las dosis adicionadas, manteniendo un rango entre 6.9 a 7.0 unidades de $\mathrm{pH}$ (remoción hasta del 85\%), este resultado es comparable con lo establecido por Guo (2008) ${ }^{45}$, quien afirma que a pH entre 3 a 11 la sábila presenta una aplicabilidad en gran cantidad de procedimientos en el ámbito del tratamiento de aguas, permitiendo porcentajes de remoción de turbidez de hasta un 92\%, lo cual se puede comparar con los resultados de Babora, et al. ( 2014) (6 $^{46}$ quienes obtuvieron que a pH altos se logran remociones de hasta un $72 \%$.

El porcentaje de remoción máxima con el extracto de naranja fue de 69\%, que en comparación con lo reportado por Ticona $(2018)^{47}$, quien realizo igualmente una extracción en base salina, logro una remoción de hasta un $81.99 \%$, cabe señalar que dicho trabajo fue realizado con valores de turbidez inicial de 13.6 NTU, lo que permite inferir sobre cómo se puede lograr mejorar los efectos de dicho extracto en relación con la turbidez inicial y la salinidad, relacionándolo con lo que obtuvo Campo, et al. ( 2018 ${ }^{48}$ de extracto de naranja en solución acuosa que removió solo un 39\% y un 79\% en combinación de quitosano. Por otra parte Contreras, et al. (2015) ${ }^{49}$, determinaron que la actividad coagulante también se puede relacionar con el ácido galacturónico, el cual también se encuentra presente en la pectina de los extractos de naranja ${ }^{50}$ y permite una mayor absorción química entre partículas suspendidas en el agua. Por su parte Miller, et al. (2008) ${ }^{51}$ lograron reducción de turbidez del 50\% en combinación con otros azucares como la galactosa, mientras que Carpio $(2007)^{52}$ logro una biosorción de Plomo $(\mathrm{Pb})$ usando extractos de naranja con una eficiencia de hasta un $95 \%$.

La estabilidad del pH luego del proceso de coagulación con el extracto de naranja y sus valores tendientes a la neutralidad contrastan con lo reportado por Chávez (2009) $)^{50}$, quién encontró un mayor rendimiento de la pectina (propia del extracto de naranja) a valores de $\mathrm{pH}$ entre 1,5 y 2.

Lo obtenido en el presente estudio, presenta entonces, un beneficio en cuanto al tratamiento de aguas crudas, puesto que las cascaras utilizadas para la extracción de coagulante, se encontraban en etapa madura, lo cual en relación con los antecedentes químicos de la naranja se asocia con un aumento de las azucares presentes en la misma y una disminución en la acidez natural de dicho fruto ${ }^{53}$.

La conductividad del agua posterior al tratamiento de coagulación con los extractos de maíz, sábila y naranja, presentaron un aumento directamente proporcional a la dosis aplicada de coagulante, esto debido a que la solución se preparó en base salina con cloruro de sodio ( $\mathrm{NaCl}$ ), que permite una mayor actividad coagulante debido a su capacidad electrolítica ${ }^{24}$. Dicha solución permitió separar las moléculas en iones de $\mathrm{Na}+$ y Cl-, logrando conducir más electricidad y al mismo tiempo permitir la precipitación de otros iones que pueden causar efectos adversos en el tratamiento de agua ${ }^{54}$.

En la resolución 2115 de $2007^{55}$ se establecen los límites máximos permisibles de turbidez (<2 NTU), conductividad $(1000 \mu \mathrm{S} / \mathrm{cm})$ y pH (entre 6.5 y 9,0) para agua de consumo humano. La conductividad en las dosis optimas de coagulante natural determinadas por su capacidad de remoción de turbidez para el maíz y sábila fueron respectivamente 512 y 371 ( $\mu \mathrm{S} / \mathrm{cm})$ lo que les da un valor agregado para ser utilizados en el tratamiento del agua, mientras que para el caso de la naranja la dosis optima presento un valor superior al de la norma $(1246 \mu \mathrm{S} / \mathrm{cm})$, por lo tanto se debe considerar su uso, debido a que se requeriría un tratamiento previo antes de ser puesta a disposición para consumo o considerar el aumento de las proporciones iniciales del componente activo adicionado en las soluciones para impedir los excesivo valores en la conductividad.

Con los valores de turbidez obtenidos luego de la aplicación de los coagulantes naturales en el agua cruda, no se logra llegar hasta los valores requeridos para consumo humano, pero cabe resaltar que este tratamiento se realiza previo a los procesos de filtración, donde probablemente se lograría obtener los valores demandados en la norma con relación a dicha variable.

Por otra parte, los extractos no alteraron significativamente los valores iniciales de $\mathrm{pH}$, estos resultados también han sido obtenidos en trabajos donde se toma como beneficio la estabilidad de dicha variable, para ser utilizados como coadyuvantes en el tratamiento de coagulación, como lo encontró Kopytko, et al. $(2014)^{56}$, donde se logró reducir en un $20 \%$ la cantidad de sulfato de aluminio requerido para tratamiento del agua, a lo que además se le suma la actividad antimicrobiana que los extractos a base del gel o cristal de la hoja de Aloe vera presentan para remover bacterias presentes en el agua ${ }^{57}$. Shahriari, et al. $(2011)^{58}$ utilizaron extractos de almidón con cloruro férrico para remover turbidez en agua preparada, logrando remociones de hasta un $92.4 \%$ y tal como encontraron Laines, et al. ( 2008)59 que se pueden utilizar extractos a base de almidón y sulfato de aluminio para ser utilizados inclusive en la remoción de turbidez de Lixiviados, logrando remociones de hasta un 98.6\%.

En contraste con los coagulantes químicos convencionales especialmente los utilizados a base de aluminio y hierro, han demostrado que al ser adicionados en el proceso de coagulación, generan variaciones en el pH óptimo (6,5 a 9,0 unidades de pH), lo que dificultan el tratamiento del agua ${ }^{60}$ acidificándola debido a la reacción que ocurre con la alcalinidad ${ }^{37}$, además, pueden generar efectos secundarios en otras variables fisicoquímicas como la turbidez y alcalinidad ${ }^{61}$, por lo que se establece que si dicha variable se encuentra en un rango de 6 y 8 unidades de $\mathrm{pH}$ las sales de aluminio se solubilizan más fácilmente, mientras que las sales de hierro permiten se utilizadas en un rango de $\mathrm{pH}$ mayor entre 5.5 y 8.5 unidades de $\mathrm{pH}$; este último compuesto permiten la formación de floc con mayor peso disminuyendo el tiempo de sedimentación pero son poco utilizadas por adicionar color al agua ${ }^{62}$.

Finalmente, en relación con lo obtenido en el presente trabajo y los posibles usos de los extractos naturales como coadyuvantes en el tratamiento de aguas con sulfato de aluminio y cloruro férrico, los cuales se encuentran actualmente regulados por normativa colombiana ${ }^{55}$ y teniendo en cuenta los estudios realizados por World Health Organization (2003) ${ }^{12}$ y Llopis y Ballester (2002) ${ }^{61}$ donde demuestran que la exposición al aluminio puede generar daños irreversibles en el sistema inmune humano e incluso permitir el desarrollo de enfermedades asociadas a daños cerebrales que afectan las capacidades psíquicas y cognitivas, se resalta el aporte que se puede dar al utilizar extractos 
de fuentes vegetales e incluso animales, enfocado en soluciones para Colombia así como lo explica Castellanos (2017)64, los que además tienen un grado de toxicidad bajo, buena biodegradabilidad y poca variabilidad del $\mathrm{pH}^{37}$, presentan un rango de dosis efectiva más amplio para la floculación de varias suspensiones coloidales y producen lodos inocuos y una menor cantidad de los mismo ${ }^{14,16}$.

\section{Conclusiones}

La presente investigación permitió realizar el proceso de coagulación-floculación con extractos de origen natural mediante la metodología del test de jarras, con lo cual se logró generar un equilibrio químico que permitió desestabilizar las cargas iónicas de los coloides favoreciendo la formación de flocs, obteniendo en este proceso una reducción de hasta el 85\% en términos de turbiedad.

La metodología empleada para la extracción de coagulantes naturales de Zea mays (Maíz), Aloe Vera (Sábila) y Citrus Sinensis (Naranja) permitió demostrar la efectividad de los mismos en la remoción de turbidez con dosis bajas de coagulante aplicado, siendo también significativo el hecho de que el $\mathrm{pH}$ del agua no se vio afectado. Aunque la remoción dada no logra satisfacer lo establecido en la normatividad colombiana de agua potable solo con el proceso de coagulación-floculación y sedimentación; no obstante, los valores ideales de turbidez podrían ser alcanzados mediante los procesos siguientes en el tren de tratamiento mediante los procesos de filtración. Adicionalmente se puede considerar el aumento del potencial de los extractos naturales al ser utilizados como coadyudantes con los coagulantes químicos comerciales. Es importante mencionar lo establecido en los objetivos de desarrollo sostenible, específicamente para Colombia en el ámbito de garantizar agua potable en el territorio nacional, por lo tanto, la alternativa estudiada puede ser tenida en cuanta como una adaptación para mitigar los problemas de salud pública y calidad de vida que se presentan actualmente.

\section{Agradecimientos}

Los autores agradecen el apoyo durante todo el proceso del proyecto al Grupo de investigación Limnología y Recursos Hídricos y sus semilleros asociados; y a la Universidad Católica de Oriente.

\section{Conflicto de interés}

Los autores declaran no tener conflicto de intereses.

\section{Referencias bibliográficas}

1. UNICEF. (2019). Agua, Saneamiento e higiene. UNICEF/ UN0215532/Rich

2. Echeverría, J., \& Anaya-Morales, S. (2018). El derecho humano al agua potable en Colombia: Decisiones del estado y de los particulares. Pontificia Universidad Javeriana.

3. UNESCO. (2019). Informe Mundial de las Naciones Unidas sobre el Desarrollo de los Recursos Hídricos. Ginebra.

4. Motta, R. (2011). El derecho al agua potable en la jurisprudencia colombiana. Revista Republicana.

5. Zamudio, C. (2012). Gobernabilidad sobre el recurso hídrico en Colombia: entre avances y retos. Revista Gest. Ambient., 15, 3, 99-11.

6. MINVIVIENDA. (2018). Plan director agua y saneamiento básico visión estratégica 2018 - 2030. Bogotá D.C

7. IDEAM. (2019). Estudio Nacional del agua 2018. Ideam, 452 pp.
8. Fuentes, L., Contreras, W., Perozo, R., Mendoza, I., \& Villegas, Z. (2008). Uso del quitosano obtenido de Litopenaeus schmitti (Decapoda, Penaeidae) en el tratamiento de agua para consumo humano. Multicias, vol 8.

9. Luna J y Benjumea Hoyos C. (2019). Evaluation of the decomposition of digital images, for indirect estimation of turbidity in water samples. Revista Bionatura 4(2) 861-871.

10. Rodriguez, J., Rojas, A., Lugo, L., \& Malaver, C. (2007). Evaluación del Proceso de la Coagulación para el Diseño de una Planta Potabilizadora. Umbral Científico, 8-16.

11. Shak, K., \& Wu, T. (2014). Coagulation-flocculation treatment of high-strengthatgroindustrial wastewater using natural Cassia obtusifolia seed gum; treat mentefficiencies and flocs characterization.

12. World Health Organization. (囚2003)凶. Aluminium in drinking-water: background document for development of WHO Guidelines for drinking-water quality. World Health Organization. https://apps.who. int/iris/handle/10665/75362.

13. Binayke, R. A., \& Jadhav, M. V. (2013). Application of Natural Coagulants in Water Purification. International Journal of Advanced Technology in Civil Engineering, 2, 118-123.

14. Guzmán, L., Villabona, Á., Tejada, C., \& García, R. (2013). Reducción De La Turbidez Del Agua Usando Coagulantes Naturales: Una Revisión. Revista U.D.C.A Actualidad \& Divulgación Científica, vol. 16. scielo.

15. WWAP. (2019). Informe Mundial de las Naciones Unidas sobre el Desarrollo de los Recursos Hídricos 2019: No dejar a nadie atrás. París: Programa Mundial de Evaluación de los Recursos Hídricos de la UNESCO.

16. Cabrera, N., Hernández, A., Simancas, E., Ayala, J., \& Almanza, K. (2017). Coagulantes naturales extraídos de lpomoea incarnata en el tratamiento de aguas residuales industriales en Cartagena de Indias. Cartagena: Sci. Tech., vol. 22.

17. Arias, A., Hernández, J., Castro, A., \& Sánchez, N. (2017). Tratamiento De Aguas Residuales De Una Central De Sacrificio: Uso Del Polvo De La Semilla De La M. Oleífera Como Coagulante Natural. Biotecnol. en el Sector Agropecuario. y Agroindustrial, vol. 15.

18. Tarón, A., Guzmán, L., \& Barros, I. (2017). Evaluación de la Cassia fistula como coagulante natural en el tratamiento primario de aguas residuales. ORINOQUIA, vol. 21.

19. Murillo Castaño, D. M. (2011). Análisis de la influencia de dos materias primas coagulantes en el aluminio residual del agua tratada. Pereira: Doctoral dissertation, Universidad Tecnológica de Pereira. Facultad de Tecnologías. Química Industrial.

20. Hildebrando Ramírez Arcila, J. J. (2015). Agentes naturales como alternativa para el tratamiento del agua. Universidad Militar Nueva Granada, 13-18.

21. Turriago, F. A., \& Melo, G. R. (2012). Evaluación de la eficiencia de la utilización de semillas de moringa oleífera como una alternativa de biorremediación en la purificación de aguas superficiales del caño cola de pato ubicado en el sector rural del municipio de Acacias. Villavicencio: Universidad nacional abierta y a distancia UNAD.

22. Sandoval A, M. M., \& Laines C, J. R. (2013). Moringa oleifera una alternativa para sustituir coagulantes metálicos en el tratamiento de aguas superficiales. Ingeniería, 17,93-101.

23. Jodi, M. L., Birnin-Yauri, U. A., Yahaya, Y., \& Sokoto, M. A. (2012). The use of some plants in water purification. Global Advanced Research Journal of Chemistry and Material Science, 4, 71-75.

24. Bravo G, M. A. (2017). Coagulantes y floculantes naturales usados en la reducción de turbidez, sólidos suspendidos, colorantes y metales pesados en aguas residuales. Bogotá D.C: Universidad Distrital Francisco José De Caldas.

25. Caicedo, J. V. (2017). Evaluación de la eficiencia como coagulante de la semilla de soja molida, soja deslipidificada y la torta de soja, frente al sulfato de aluminio en procesos de clarificación de aguas. Universidad Distrital Francisco José De Caldas, 7-8.

26. Domínguez F, R., Arzate, I., Chanona, J., Welti, J., Alvarado, J., Calderón, G., .. . Gutiérrez, G. (2012). El ger de Aloe vera: estructura, composición química, procesamiento, actividad biológica e importancia en la industria farmacéutica y alimentaria. Revista Mexicana de Ingeniería Química, 11, 23-43. 
27. Garcia, B. (2007). Metodología de extracción in situ de coagulantes naturales para la clarificación de agua superficial. Aplicación en países en vías de desarrollo. Universidad politécnica de valencia, 23-30.

28. Aguirre, S. E., Piraneque, N. V., \& Cruz, R. K. (2018). Sustancias naturales: Alternativa para el tratamiento de agua del río Magdalena Palermo Colombia. Scielo, 29, n. 3.

29. ASTM, D.-8. (2003). Standard Practice for Coagulation-Flocculation Jar Test of Water, ASTM International. West Conshohocken: ASTM International.

30. Lilliefors, H. W. (1967). On the Kolmogorov-Smirnov test for normality with mean and variance unknown. Journal of the American statistical Association, 62(318), 399-402.

31. Shapiro, S. S., \& Wilk, M. (1965). An analysis of variance test for normality (complete samples). Biometrika, 591-611.

32. Levene, Howard (1960). Robust tests for equality of variances. En Ingram Olkin, Harold Hotelling, et al., ed. Stanford University Press. pp. 278-292.

33. Guisande C., Heine J., González-DaCosta J. \& García-Roselló (2014). RWizard Software. University of Vigo. Vigo, Spain.

34. Shogren, R. L. (2009). Flocculation of kaolin by waxy maize starch phosphates. Carbohydrate Polymers, (76) 639-644.

35. Jiménez Benavides, D. L., \& Piscal, B. V. (2015). Estudio y evaluación del almidón de maíz como alternativa natural en el proceso de coagulación de agua para consumo humano. San Juan de Pasto: Universidad de Nariño.

36. Sotheeswaran, S., Nand, V., Matakite, M., \& Kanayathu, K. (2011). Moringa oleifera and other local seeds in water purification in developing countries. Chemistry and Environment, 15

37. Trujillo, D., Duque, L. F., Arcila, J. S., Rincón, A., Pacheco, S., \& Herrera, O. F. (2014). Turbidity removal in a water sample from a natural source via coagulation/flocculation using plantain starch.

38. Hernández Curbelo, C., Moreno Quintero, M. E., Hernández Ramírez, D., \& Crespo Zafra, L. M. (2016). Acid hydrolysis of Aloe Vera (Sábila) bagasse for obtaining pectin. Centro Azúcar, 44.

39. Sánchez F., S. A., \& Unitiveros B., G. (2004). Determinación de la actividad floculante de la pectina en soluciones de Hierro (III) y Cromo (III). Sociedad Química del Perú, 4, 201-208.

40.Perazzo C, F. G., Goulart, C., Figueiredo, D., Oliveira, C., \& Silva, J. (2008). Economic and Environmental Impact of Using Exogenous Enzymes on Poultry Feeding. International Journal of Poultry Science, 7, 311-314.

41. Diestra R, F. S., \& Ramos P, I. V. (2019). Efecto de la concentración de Aloe Vera (Sábila) y tiempo de floculación en la remoción de sólidos suspendidos y materia orgánica biodegradable de aguas residuales municipales sector el Cerillo, Santiago de Chuco. Trujillo - Perú: Biblioteca Digital - Dirección de Sistemas de Informática y Comunicación.

42. Munavalli, G. (2017). Use of Aloe Vera as Coagulant aid in Turbidity Removal. Engineering Research and Technology, 10, 1.

43. Morales O, J. A. (2018). Determinación del poder coagulante de la sábila para la remoción de turbidez en el proceso de tratamiento de agua para consumo humano. Oxapampa: Universidad Nacional Daniel Alcides Carrión.

44. Mendoza, I., Fuentes, L., \& Díaz, A. (2014). Aloe barbadensis (Miller) como coagulante natural en la potabilización de aguas con baja turbidez. Zulia-Venezuela: LIANCOL.

45. Guo, H. (2008). Study on the Natural Macromolecular Flocculant of Aloe. Aloe trade.

46. Babora B, R., Freire, R., \& Oliveira B, W. (2014). Remoción de turbidez de agua usando Aloe vera como coagulante natural. Forum Ambiental, 10, 1-11.

47. Ticona, V. E. (2018). Estudio de la determinación de la actividad floculante en agua provenientes del río Chilo conteniendo $\mathrm{As}, \mathrm{Pb}$ y Cr tratados con pectina obtenidos a partir de la cáscara de naranja, limón y mandarina. Arequipa- Perú: Universidad Nacional San Agustín de Arequipa.

48. Campo Vera, Y., Delgado, M. A., Roa, Y., Mora, G., \& Carreño Ortiz, J. (2018). Preliminary evaluation of the effect of chitosan and orange peel in the coagulation-flocculation of wastewater. U.D.C.A Actualidad \& Divulgación Científica, 21, 565-572.
49. Contreras L, K. P. Mendoza A, Y. Mendoza S, J. G., Verbel O, R, \& Mendoza O, G. P. (2015). El Nopal (Opuntia ficus-indica) como coagulante natural complementario en la clarificación de agua. Producción más limpia, 10, 40-50.

50. Chávez Milla, J. M. (2009). Extraction of pectin from peel "criolla orange" (Citrlls aurantium L.) from Rodríguez de Mendoza Province. Investigaciones Amazonenses, 3, 24-26.

51. Miller, S. M., Fugate, E. J., $\quad$ Craver O, V., Smith, J. A., \& Zimmerman, J. B. (2008). Toward Understanding the efficacy and Mechanism of Opuntia spp. as a Natural Coagulant for Potential Application in Water Treatment. Environmental Science \& Technology, 42, 42744279 .

52. Carpio M, J. C. (2007). Biosorción de plomo (II) por cáscara de naranja "citrus cinensis" pretratada. ResearchGate, Lima- Perú.

53. Agustí M. (2003). Citricultura. Madrid-México: Mundi-Prensa Libros.

54. Arango-Ruiz, Á. (2012). Effects of the $\mathrm{pH}$ and the conductivity on the electrocoagulation of waste water from dairy industries.

55. MINVIVIENDA (2007). Resolución no 2115 de 22 junio de 2007. Por medio de la cual se señalan características, instrumentos básicos y frecuencias del sistema de control y vigilancia para la calidad del agua para consumo humano.

56. Kopytko, M. I., Villamizar R, E. P., \& Picón R, Y. (2014). Application of Natural Product (Aloe Vera) in coagulation-Flocculation procedures for Water Treatability Study. International Journal of Engineering Science and Innovative Technology, III.

57. Musmeci, R., \& Lezcano, M. T. (2013). Acción antimicrobiana del gel de aloe vera sobre staphylococcus aureus, escherichia coli, pseudomonas aeruginosa y candida albicans. Revista sobre Estudios e Investigaciones del Saber Académico, 23-27.

58. Shahriari, T., Nabi Bidhendi, G., \& Shahriari, S. (2011). Evaluating the Efficiency of Plantago Ovata and Starch in Water turbidity removal. International Journal of Environmental Research and Public Health, 6, 259-264

59. Laines C, J. R., Goñi A, J. A., Schroeder A, R. H., \& Camacho C, W. (2008). Mezcla con potencial coagulante para tratamiento de lixiviados de un relleno sanitario. Interciencia, 33, 22-28.

60. Cogollo, J. M. (2011). Water clarification using polymerized coagulants: Aluminum Hydroxychloride case. Dyna, 78, 18-27.

61. Llopis S, L., \& Ballester D, F. (2002). Revisión de los estudios sobre exposición al aluminio y enfermedad de Alzheimer. Salud Pública, 76, 645-658.

62. Restrepo, H. A. (2009). Evaluación del proceso de coagulación-Floculación de una planta de tratamiento de agua potable. Medellín: Universidad Nacional de Colombia.

63. Castellanos P, F. L. (2017). Revision of the use of natural coagulants in the process of water clarification in Colombia. Bogotá D.C: Universidad Militar Nueva Granada.

64. Tukey, J. (1949). Comparing Individual Means in the Analysis of Variance. Biometrics, 5 (2), 99-114

Received: 15 febrero 2021

Accepted: 15 marzo 2021 